Research Article

\title{
Online Dynamic Tip-Over Analysis for a Wheeled Mobile Dual-Arm Robot with an Improved Tip-Over Moment Stability Criterion
}

\author{
Xianhua Li $\mathbb{D},{ }^{1}$ Liang Wu $\mathbb{D}^{1},{ }^{1}$ Qing Sun $\mathbb{D}^{2},{ }^{2}$ and Tao Song ${ }^{2,3}$ \\ ${ }^{1}$ School of Mechanical Engineering, Anhui University of Science and Technology, Huainan 232001, China \\ ${ }^{2}$ School of Mechatronics Engineering and Automation, Shanghai University, Shanghai 200444, China \\ ${ }^{3}$ Shanghai Robot Industry Technology Research Institute Co., Ltd., Shanghai 200063, China
}

Correspondence should be addressed to Xianhua Li; xhli01@163.com

Received 29 March 2021; Revised 6 June 2021; Accepted 25 June 2021; Published 12 July 2021

Academic Editor: Weitian Wang

Copyright (c) 2021 Xianhua Li et al. This is an open access article distributed under the Creative Commons Attribution License, which permits unrestricted use, distribution, and reproduction in any medium, provided the original work is properly cited.

Tip-over stability analysis is critical for the success of mobile manipulation of the dual arm, especially in the cases that the dual arm or the mobile platform moves rapidly. Due to strong dynamics coupling between the dual arm and mobile platform, online evaluation of dynamic stability of the mobile dual-arm robot still remains challenging. This paper presents an improved tip-over moment stability criterion dealing with the dual arm and mobile platform interaction and proposes an algorithm for calculating the tip-over stability margin of the arm end in the workspace to analyze the dynamic stability of the wheeled mobile dual-arm robot. The simulations on a four-wheeled mobile dual-arm robot validate the correctness and feasibility of the proposed method.

\section{Introduction}

The wheeled mobile dual-arm robot (WMDAR) is an emerging class of robots which have capabilities of both moving and manipulation. The WMDAR is usually humanrobot collaboration, enabling it to be widely employed in home service, restaurant service, and medical treatment [1-3]. However, the WMDAR is a kind of system with unstable structure, which may tip-over under the action of different dynamic factors or external interference, especially for the mobile service robot with small mobile platform, large variation of system mass distribution, and bearing extraload in work.

Currently, some tip-over stability criteria have been applied in mobile robots [4-9]. Among the many stability criteria, the zero moment point (ZMP) is the most popular. Sugano et al. presented concepts of the stability degree and the valid stable region based on the zero moment point (ZMP) [7]. Korayem et al. proposed an algorithm for determining the maximum load carrying capacity of a mobile manipulator considering tip-over stability based on the ZMP for the obstacle environment [8]. Kagami et al. [9] described a fast dynamically equilibrated trajectory generation method for a humanoid robot based on the relationship between the robot's center of gravity and the ZMP. However, if the center of mass of the robot system changes, ZMP is not sensitive to the stability of the system. Therefore, Papadopoulos and Rey defined the tipping stability margin according to the forceangle (FA) margin criterion and described a real-time rollover prediction and prevention scheme based on static and dynamic force-angle measure $[10,11]$. This criterion ignores the reaction force and moment of the manipulator acting on the moving platform. Then, Moosavian and Alipour proposed a moment-height stability (MHS) measure for the wheeled mobile robot considering robot dynamics and system gravity center [12-15]. In addition, some other stability criteria were applied to the stability detection of the mobile manipulator. Ghassempoor and Sepehri proposed a method to measure the stability according to the energy level of the moment acting on the support boundary [16]. A method was presented for tip-over stability analysis of a wheeled mobile manipulator based on tip-over moment by 
Guo et al. [17] The normal bearing force criterion [18] requires force sensors to measure the bearing force, which is costly. At present, many works have been performed on the tip-over stability criterion of the mobile robot, but, at the same time, some papers focus on the mobile robot tip-over avoidance algorithm.

Moubarak and Ben-Tzvi [19] proposed a global optimal attitude convergence algorithm for redundant serial robots, which can prevent tipping without considering the influence of joint velocity and acceleration on tipping stability. Rey and Papadopoulos [11] used the FA measure method for initial configuration of the robot to avoid the robot tipping over. Based on an adaptive neural fuzzy algorithm, Li and Liu [20] utilized self-motions of redundant mobile manipulators to improve a robot's stability. Ding et al. [21] proposed a real-time tipping avoidance algorithm to reduce the transmission of tipping torque by adjusting the manipulator posture or changing the robot speed, which can effectively avoid the robot tipping. Many environments and scenarios contain rough and irregular terrain and are difficult for robots. Agheli and Nestinger [22] presented a multilegged reactive stability control method for maintaining system stability under external perturbations. Feng et al. [23] introduced a new method for evaluating the stability of robots in rugged terrain and proposed an algorithm for automatically realizing self-balancing of robots. Kashyap and Parhi [24] utilized the LIPM plus flywheel model (LIPPFM) for analysis of the complete dynamic motion of the humanoid robot. It can be seen that the previous research on the stability maintenance of the robot motion process mainly focused on the attitude change of the manipulator of the single-arm mobile robot through the analysis of the above literature. However, there is no research on the attitude change of the two arms and the overturning compensation caused by the speed and acceleration of the two arms.

In this paper, an improved tip-over moment stability criterion is proposed dealing with dual-arm and mobile platform interaction. Meanwhile, an algorithm for calculating the tip-over stability margin of manipulator workspace is also presented to analyze the dynamic stability of WMDAR. This paper proposes a method to study the stability of WMDAR, and this algorithm is very important for the follow-up research. The dynamic stability of the robot can be studied by integrating the algorithm into the control elements, which lays a foundation for the trajectory planning of the robot to tip-over avoidance.

This paper is divided into six sections. The kinematics and force model of this robot are analyzed in Section 2. An improved tip-over moment stability criterion is presented in Section 3. Then, in Section 4, the workspace pose discretization is solved and an algorithm for calculating the tipover stability margin of the manipulator is proposed. In Section 5, simulation in MATLAB software is carried out in order to validate correctness of this improved tip-over stability criterion and an algorithm for calculating the tipover stability margin of workspace to analyze the stability of WMDAR is proposed, and in Section 6, some conclusions on them are drawn.

\section{Kinematic and Force Model of the Wheeled Mobile Dual-Arm Robot}

2.1. Kinematic Model of the Wheeled Mobile Dual-Arm Robot. The WMDAR consists of a four-wheel mobile platform, a waist, and the dual arm mounted on the mobile platform, depicted in Figure 1. The four-wheel mobile platform is composed of a platform, two driving wheels, and two driven wheels, in which two driving wheels go forward or turn through differential drive.

To describe the motion of the WMDAR, coordinate systems were established, i.e., the world frame $O_{W} X_{W} Y_{W} Z_{W}$, the robot body frame $O_{S} X_{S} Y_{S} Z_{S}$, the left arm frame $O_{L} X_{L} Y_{L} Z_{L}$, the end frame of left arm $O_{L L} X_{L L} Y_{L L} Z_{L L}$, the right arm $O_{R} X_{R} Y_{R} Z_{R}$, and the end frame of right arm $O_{R R} X_{R R} Y_{R R} Z_{R R}$, as shown in Figure 2(a). And, $m$ is the mass of the mobile platform and the body, and $O_{C}$ is the center of mass of the mobile platform and the body. In the four-wheel mobile platform, points $p_{1}, p_{2}, p_{3}$, and $p_{4}$ are the contact points between the mobile platform and the ground and the four black solid lines connecting the adjacent two points are the four tip-over axes of the robot system, as shown in Figure 2(b). Meanwhile, the relevant parameters of the robot system are shown in Table 1, and the position parameters of the point in the table are expressed in the reference coordinate frame $O_{S} X_{S} Y_{S} Z_{S}$.

Then, the transformation matrices between different frames are given as follows, and the pose of the end of the arm could be obtained by the screw theory [25], as shown in equation (3):

$$
\begin{aligned}
{ }_{\mathbf{S}}^{\mathbf{W}} \mathbf{T} & =\left[\begin{array}{cccc}
\cos \theta_{m} & -\sin \theta_{m} & 0 & d_{x m} \\
\sin \theta_{m} & \cos \theta_{m} & 0 & d_{y m} \\
0 & 0 & 1 & 0 \\
0 & 0 & 0 & 1
\end{array}\right], \\
{ }_{\mathbf{L}} \mathbf{T} & =\left[\begin{array}{cccc}
\cos \theta_{l} & 0 & \sin \theta_{l} & 0 \\
0 & 1 & 0 & \frac{L}{2} \\
-\sin \theta_{l} & 0 & \cos \theta_{l} & d \\
0 & 0 & 0 & 1
\end{array}\right], \\
\mathbf{s}_{\mathbf{r}} \mathbf{T} & =\left[\begin{array}{cccc}
\cos \theta_{l} & 0 & \sin \theta_{l} & 0 \\
0 & 1 & 0 & -\frac{L}{2} \\
-\sin \theta_{l} & 0 & \cos \theta_{l} & d \\
0 & 0 & 0 & 1
\end{array}\right], \\
g_{s t}(\theta)= & \widehat{\widehat{\xi}}^{\hat{\xi}_{1} \theta_{1}}, \ldots, e^{\hat{\xi}_{6} \theta_{6}} g_{s t}(0)=\left[\begin{array}{cccc}
n_{x} & o_{x} & a_{x} & p_{x} \\
n_{y} & o_{y} & a_{y} & p_{y} \\
n_{z} & o_{z} & a_{z} & p_{z} \\
0 & 0 & 0 & 1
\end{array}\right] .
\end{aligned}
$$

For the sake of simplification of analysis and computations conducted in the paper, the following assumptions are made:

(1) The ground is even, and no surface shrinkage is considered 

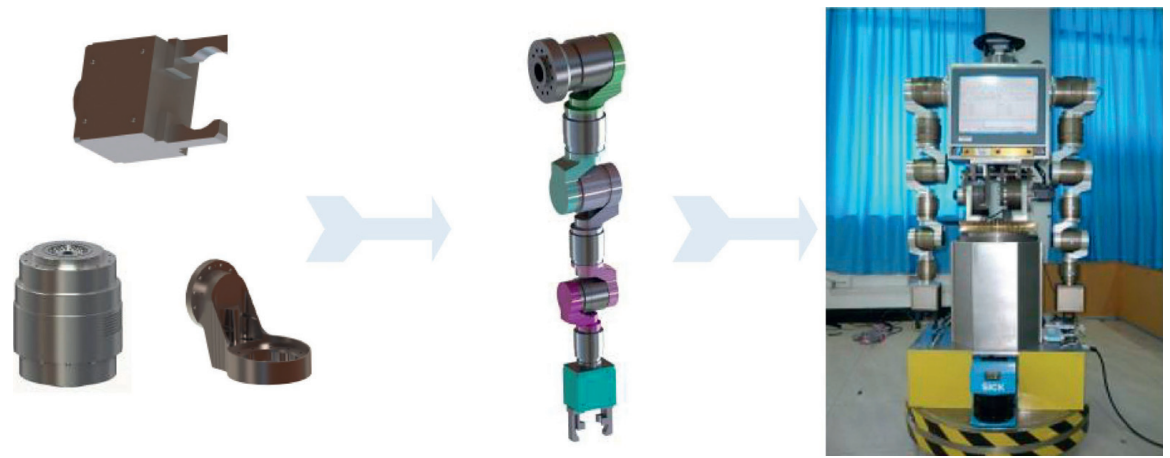

Figure 1: Model of the wheeled mobile dual-arm robot.

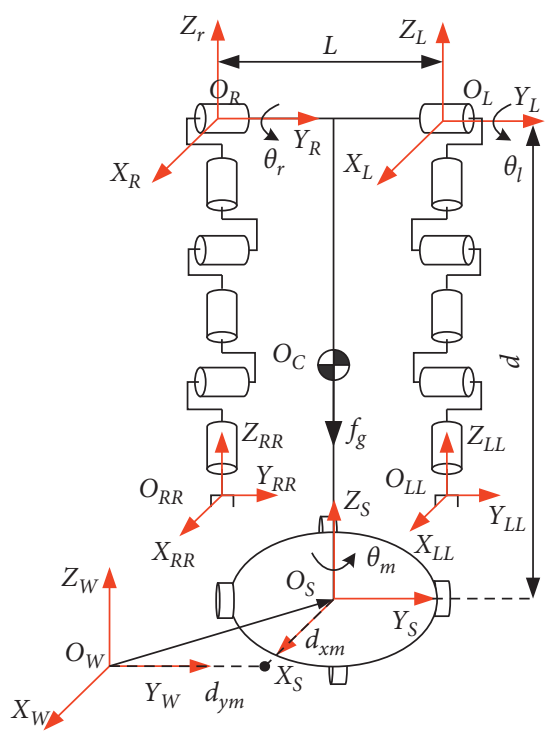

(a)

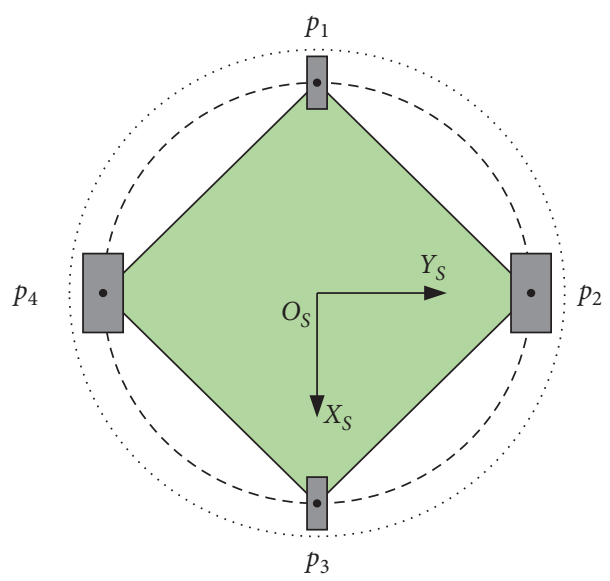

(b)

Figure 2: Coordinates' system definition. (a) Dual-arm robot system. (b) Platform system.

TABLE 1: Related parameters of the robot system.

\begin{tabular}{lc}
\hline Parameters & Values \\
\hline$p_{1}(\mathrm{~m})$ & {$[0.604,0,0]$} \\
$p_{2}(\mathrm{~m})$ & {$[0,0.604,0]$} \\
$p_{3}(\mathrm{~m})$ & {$[-0.604,0,0]$} \\
$p_{4}(\mathrm{~m})$ & {$[0,-0.604,0]$} \\
$O_{r}(\mathrm{~m})$ & {$[0,-0.399,1.278]$} \\
$O_{l}(\mathrm{~m})$ & {$[0,0.399,1.278]$} \\
$O_{c}(\mathrm{~m})$ & {$[0,0,0.0658]$} \\
$m(\mathrm{~kg})$ & 60 \\
\hline
\end{tabular}

(2) All wheels are always in point contact with the ground, i.e., no slippage of the wheels occurs

(3) The dual arm and body are rigidly connected with the platform, and the links and joints of the manipulator are rigid

(4) The mobile platform and the body were taken as a whole because the influence of the robot's body motion is not considered in this paper.

2.2. Force Model of the Wheeled Mobile Dual-Arm Robot. In this paper, the dynamic model was mainly aimed at the dynamic model of the manipulator. The force/moment of the arm end to joint 1 can be obtained through the iterative relationship between the links based on the Newton Euler method and screw theory.

2.2.1. The Speed of Link. The Jacobian matrix of each link can be obtained by combining the Newton Euler method and screw theory, as shown in the following equation:

$$
\mathbf{J}_{i}=\left[A d_{e^{\hat{\xi}_{1} \theta_{1}} \xi_{1}} \xi_{1}, \ldots, A d_{e^{\hat{\xi}_{i} \theta_{i}}} \xi_{i}, \ldots, 0\right], \quad i=1,2, \ldots, n .
$$

Therefore, the relationship between the speed of each joint and the speed of the $i$ th link was

$$
V_{i}=\mathbf{J}_{i} \dot{\theta} .
$$

2.2.2. The Acceleration of Link. The acceleration of each link can be calculated by deriving the following equation:

$$
\dot{V}=\mathbf{J}_{i} \ddot{\theta}+\dot{J}_{i} \dot{\theta},
$$

where $\dot{J}_{i}=\left[V_{0} \times A d \widehat{e^{\xi_{1} \theta_{1}}} \xi_{1}, \ldots, V_{i-1} \times A d \widehat{e^{\xi_{i} \theta_{i}}} \xi_{i}, \ldots, 0\right], i=$ $1,2, \ldots, n$, where $V_{0}$ is the velocity of the base (body and mobile platform) in equation (5). 
2.2.3. Force/Moment Equation of Link. The force balance equation of $i$ th link can be obtained, as shown in the following equation:

$$
w_{i}=w_{i}^{J}-w_{i+1}^{J}+w_{i}^{G}+w_{i}^{E}=I_{i} \dot{V}+V_{i} \times{ }^{*} I_{i} V
$$

where $w_{i}^{\mathrm{J}}$ is the generalized joint force/moment produced by the $i$ th joint, $w_{i+1}^{J}$ is the generalized joint force/moment produced by the joint $i+1$ exerted on link $i, w_{i}^{G}$ is the force/ moment of gravity exerted on link $i, w_{i}^{E}$ is the sum of other external forces/moment, $I_{i}=A d_{e^{\xi_{i} \theta_{i}}}^{*} I_{i 0}\left(A d_{e^{\xi_{i} \theta_{i}}}^{*}\right)^{T}, I_{i}$ is the space inertia of the current manipulator configuration, and $I_{i 0}$ is the spatial inertia of the link $i$ in the initial configuration of the manipulator. The above formula was derived from the arm base coordinate frame (for example, coordinate frame $O_{L} X_{L} Y_{L} Z_{L}$ for left arm). The force/moment balance equation of joint $i$ can be obtained by combining the Newton Euler method and screw theory, as shown in the following equation:

$$
w_{i}^{\mathrm{J}}=w_{i+1}^{\mathrm{J}}-w_{i}^{G}-w_{i}^{E}+I_{i} \dot{V}+V_{i} \times^{*} I_{i} V .
$$

Equation (8) provides a reverse iterative method to calculate the joint constraint force/moment, which can be calculated from the end effector to the last joint $n$ of the arm until joint 1 . The constraint force/moment on joint 1 and the force/moment of the arm acting on the body and mobile platform are reciprocal from Figure 3.

\section{Improved Tip-Over Moment Stability Criterion}

In this section, we have derived a new tip-over moment stability criterion for WMDAR with consideration of dual arm-mobile platform interactions. Figure 3 depicts the various forces and moment exerting on the body and mobile platform. The reaction wrench from the arm onto the body and mobile platform is expressed as $-w_{1}$ based on Section

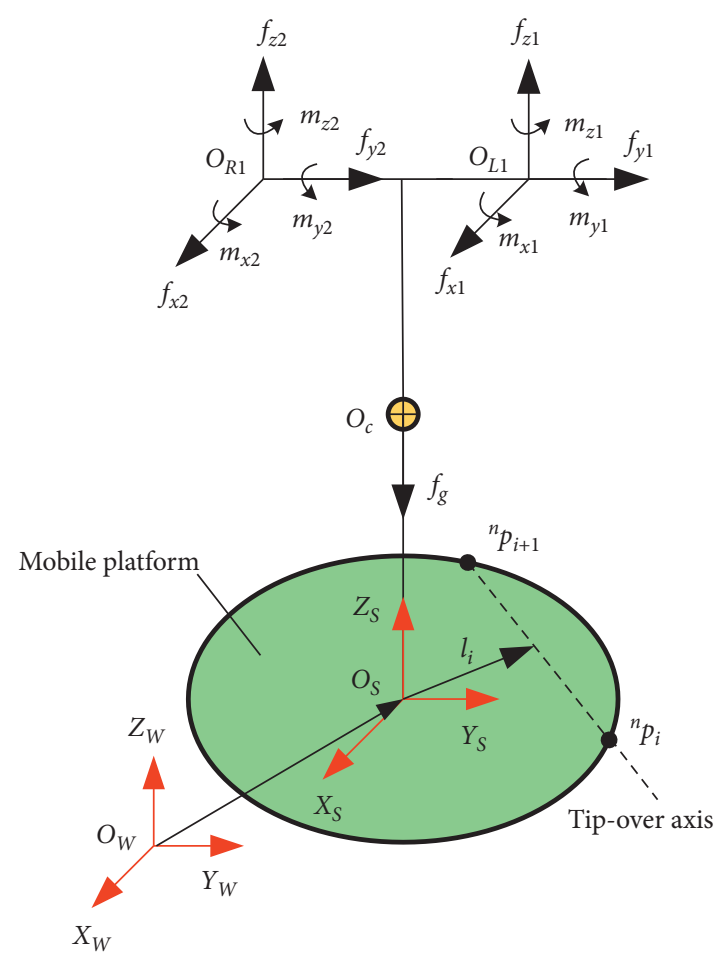

FIgURE 3: Force and moment model of the WMDAR.

2.2, and the force/moment consists of components in three directions, as shown in the following equation:

$$
\begin{aligned}
&-w_{1}=\left[\begin{array}{ll}
f_{M}^{T}, m_{M}^{T}
\end{array}\right]^{T}, \\
&\left\{\begin{array}{lll}
f_{M} & =\left[\begin{array}{lll}
f_{1 x} & f_{1 y} & f_{1 z}
\end{array}\right]^{T}, \\
m_{M} & =\left[\begin{array}{lll}
m_{1 x} & m_{1 y} & m_{1 z}
\end{array}\right]^{T} .
\end{array}\right.
\end{aligned}
$$

The tip-over moment (TOM) on the tip-over axis (TOA) $\mathbf{a}_{i i+1}$ can be calculated through the above calculation:

$$
\mathrm{TOM}_{i}=\left(f_{g} \times l_{i}\right) \cdot \mathbf{a}_{\mathbf{i i}+1}+\sum_{j=1}^{k}\left[\left(m_{M} \cdot \mathbf{a}_{\mathbf{i i}+1}\right)+\left(f_{M} \times d_{i}\right) \cdot \mathbf{a}_{\mathbf{i i}+1}\right] \quad(k=1 \text { and } 2) .
$$

The first item in equation (11) is the moment of gravity exerting on the TOA of the body and mobile platform. The second item is the moment of the force and moment produced by both arms exerting on the TOA. In this paper, the robot system was divided into three modules, the body and mobile platform, the left arm, and the right arm. Therefore, $f_{g}$ denotes the gravity of the body and mobile platform. The gravity of the body and mobile platform and the force/ moment of the left or right arm exerting on the TOA for the robot system play an important role in the stability of the system.

If tipping occurs, the robot will tip over outward along the TOA formed by two adjacent wheels, where $\mathbf{a}_{\mathbf{i i}+1}$ represents a unit vector for the TOA, which can be obtained by the coordinates of two adjacent wheel-terrain contact points $\left({ }^{n} p_{i},{ }^{n} p_{i+1}\right)$, i.e.,

$$
\mathbf{a}_{\mathbf{i i}+1}=\frac{{ }^{n} p_{i+1}-{ }^{n} p_{i}}{\left|{ }^{n} p_{i+1}-{ }^{n} p_{i}\right|}
$$

According to the dynamic method of the rigid body translation, the moments of both arms and body and mobile platform relative to the TOA $\mathbf{a}_{\mathbf{i i}+1}$ were calculated. For the WMDAR, the minimum TOM exerting on TOA of the mobile platform is as follows:

$$
\mathrm{TOM}=\min \left\{\mathrm{TOM}_{1}, \mathrm{TOM}_{2}, \ldots, \mathrm{TOM}_{n}\right\} .
$$

Equation (13) indicates that when the minimum TOM of the robot system is less than 0 , that is, the TOM of the robot 
system along the TOA is outward, the robot system will tip over. Therefore, the tip-over stability margin (TOSM) is defined as

$$
\begin{aligned}
\varphi_{i} & =\frac{\mathrm{TOM}_{i}}{\mathrm{TOM}_{\text {norm }}}, \\
\varphi & =\min \left\{\varphi_{1}, \varphi_{2}, \ldots, \varphi_{n}\right\},
\end{aligned}
$$

where $\mathrm{TOM}_{\text {norm }}$ represents a constant value, which is the minimum TOM exerting on TOA when the WMDAR is in a steady state, that is, $\mathrm{TOM}_{\text {norm }}>0$. Therefore, the stability of the WMDAR system can be determined by the TOSM. When $\varphi>0$, that is, the minimum TOM on the TOA will be greater than 0 , and the WMDAR system will be stable; however, when $\varphi<0$, it means that the moment on the TOA is outward, which means that the system may tip over.

\section{Calculating TOSM in the Arm Workspace}

4.1. Position Discretization of Arm Workspace. In order to study the influence of arm motion on the tip-over stability of WMDAR, the workspace of the arm should be discretized. In this paper, we used the following method to solve the arm workspace discretization and chose the left arm to introduce the position and posture discrete method. Firstly, a sphere whose radius is the total length $L$ of the arm was established based on the coordinate system $\left(O_{L} X_{L} Y_{L} Z_{L}\right)$. Then, the radius of the sphere was divided into $N_{r}$ parts, and all spheres with radius from the coordinate origin to each bisection point were established. The radius calculation formula of all spheres is as follows:

$$
r_{s}=\frac{k L}{N_{r}} \quad\left(k=1,2, \ldots, N_{r}\right) .
$$

The distribution points on the surface of each sphere were taken, as shown in Figure 4, and all the distribution points can constitute the discrete workspace points of the arm.

Assuming that the radius of the sphere is $r_{s}$, the position of distribution points can be obtained by the following formula based on the coordinate system $\left(O_{L} X_{L} Y_{L} Z_{L}\right)$ :

$$
\begin{gathered}
\theta_{i}=\frac{\left(2 i-N_{a}-2\right) \pi}{N_{a}}, \\
\phi_{j}=\frac{\left(2 i-1-N_{b}\right) \pi}{\left(2\left(N_{b}-1\right)\right)} \\
\left\{\begin{array}{l}
x=r_{s} \cos \theta_{i} \cos \phi_{j}, \\
y=r_{s} \sin \theta_{i} \cos \phi_{j}, \\
z=r_{s} \sin \theta_{i}, \\
p_{i, j, s}=[x, y, z]^{T},
\end{array}\right.
\end{gathered}
$$

where $N_{a}$ and $N_{b}$ represent the step size of $\theta$ and $\phi$, respectively, that is to say, they indicate the density of distribution points on the surface of the sphere.

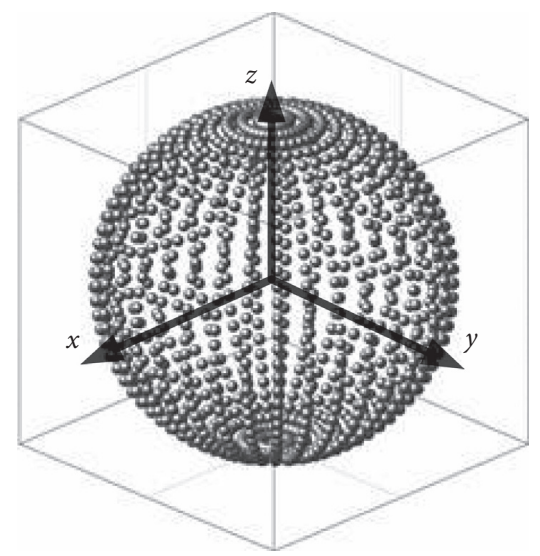

Figure 4: Discrete workspace of the manipulator.

4.2. Pose Discretization of the Arm Workspace. There are many possibilities for the pose of the end at this distribution point, which will affect the configuration of the arm. Therefore, the pose of the end was discretized to study the tip-over stability of WMDAR, in which the arm has different configuration. Firstly, a sphere with radius of 1 was established on the workspace point, and the center of the sphere coincides with the workspace point. A spherical coordinate system with the center of the sphere as the origin was established on the sphere. Then, the position of each uniform point on the surface of the sphere relative to the spherical coordinate system was solved by using the uniform distribution algorithm [26], as shown in Figure 5(a). The positions of these uniform points can be calculated by the following formula:

$$
\begin{aligned}
& \theta_{k}=\arccos \left(h_{k}\right), \\
& h_{k}=-1+\frac{2(k-1)}{N_{p}-1} \quad\left(1 \leq k \leq N_{p}\right), \\
& \beta_{k}=\left(\beta_{k-1}+\frac{3.6}{N_{p}} \frac{1}{\sqrt{1-h_{k}^{2}}}\right)(\bmod (2 \pi)) \quad\left(2 \leq k \leq N_{p}-1\right) .
\end{aligned}
$$

When $k=1$ or $k=N_{p}, \beta_{1}=\beta_{N_{p}}=0$ :

$$
\left\{\begin{array}{l}
x_{k}=\sin \beta_{k} \cos \frac{\beta_{k}}{2}, \\
y_{k}=\sin \beta_{k} \sin \frac{\beta_{k}}{2}, \\
z_{k}=\cos \frac{\beta_{k}}{2}, \\
p_{k}=\left[\begin{array}{lll}
x_{k} & y_{k} & z_{k}
\end{array}\right]^{T} .
\end{array}\right.
$$

Then, we take the direction from the sphere center to each uniform point as the $z$-axis direction of each discrete pose coordinate system, and the direction of the $x$-axis can be set arbitrarily, as shown in Figure 5(b). Therefore, the 


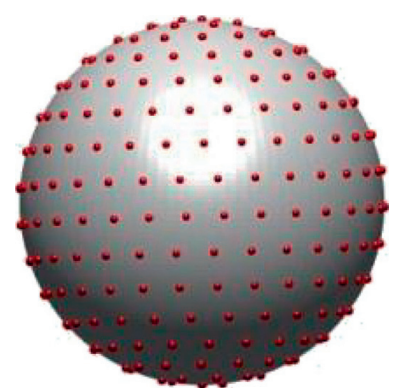

(a)

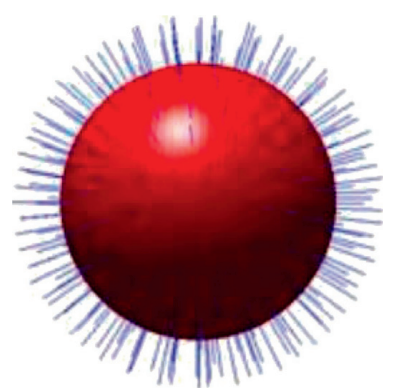

(b)
FIgURE 5: Uniform points on the discrete spheres and their $Z$-axis distribution.

pose matrix of all the discrete pose coordinate systems relative to the sphere coordinate system on a workspace point can be expressed as follows:

$$
\begin{aligned}
\mathbf{R}_{k}=\mathbf{R}_{z, \beta_{k}} \mathbf{R}_{y^{\prime}, \theta_{k}} & =\left[\begin{array}{ccc}
c_{\beta_{k}} & -s_{\beta_{k}} & 0 \\
s_{\beta_{k}} & c_{\beta_{k}} & 0 \\
0 & 0 & 1
\end{array}\right]\left[\begin{array}{ccc}
c_{\theta_{k}} & 0 & s_{\theta_{k}} \\
0 & 1 & 0 \\
-s_{\theta_{k}} & 0 & c_{\theta_{k}}
\end{array}\right] \\
& =\left[\begin{array}{cccc}
c_{\beta_{k}} c_{\theta_{k}} & -s_{\beta_{k}} & c_{\beta_{k}} s_{\theta_{k}} \\
s_{\beta_{k}} c_{\theta_{k}} & c_{\beta_{k}} & s_{\beta_{k}} s_{\theta_{k}} \\
-s_{\theta_{k}} & 0 & c_{\theta_{k}}
\end{array}\right],
\end{aligned}
$$

where $\mathbf{R}_{z, \beta_{k}}$ denotes the matrix after rotating $\beta_{k}$ around $z$ axis, $\mathbf{R}_{y^{\prime}, \theta_{k}}$ denotes the pose matrix after rotating $\theta_{k}$ around $y$-axis, and $s$ and $c$ are the abbreviations of sin and cos, respectively.

The pose matrix of each discrete pose coordinate system to the sphere coordinate system is shown as follows:

$$
\mathbf{T}_{k}=\left[\begin{array}{cc}
\mathbf{R}_{k} & 0 \\
0 & 1
\end{array}\right] .
$$

The pose matrix of all the points in the workspace relative to the base coordinate system $\left(O_{L} X_{L} Y_{L} Z_{L}\right)$ of the arm through equation (20) can be obtained:

$$
\mathbf{T}_{i, j, s, k}=\left[\begin{array}{cc}
\mathbf{R}_{k} & p_{i, j, s} \\
0 & 1
\end{array}\right] .
$$

4.3. Calculation of TOSM of the Arm Workspace. The discrete pose of a point in the workspace can be obtained by equation (25). The inverse kinematics analysis shows that the pose will correspond to eight groups of configurations of the arm when the end of the arm is in this pose (if the end cannot reach the point, there is no solution). The joint angle of each group arm configuration can be obtained according to the inverse kinematics analysis, and the force/moment of joint 1 can be obtained by arm dynamics. Then, the TOSM of each pose can be calculated based on Section 3. The TOSM in the workspace point of the arm was defined as the minimum TOSM of all configurations corresponding to all discrete poses on the workspace point was taken as the TOSM of this point:

$$
\psi=\min \left\{\varphi_{i, j}\right\}, \quad\left\{\begin{array}{l}
i=1,2, \ldots, N_{p} \\
j=1,2, \ldots, 8
\end{array}\right.
$$

The TOSM of each workspace point represents the tipover stability of the system when the end of the arm is located at the point. When $\psi>0$, it indicates that the system is always in a stable state, and the larger the value, the better the stability of the system; otherwise, when $\psi<0$, the system may tip over.

\section{Motion Analysis of Tip-Over Stability of WMDAR System}

We proposed a modular decomposition method in order to verify the correctness of algorithm calculated TOSM. Firstly, the body and mobile platform, left arm, and right arm of the robot system were divided into three modules. In this paper, we keep the right-arm module as the initial state in the simulation calculation, as shown in Figure 2(a), and the influence of the right arm on the stability of the robot system is only the gravity. At the same time, we control the movement of the left-arm module and study the influence of arm motion on the stability of the robot system.

5.1. Static Case. In this section, the tip-over stability of the robot system was studied when the left arm was in a static state in its workspace. The discrete parameters of workspace points were selected as follows: $N_{r}=20, N_{a}=31$, and $N_{b}=31$. The joint velocity and acceleration of the arm were both 0 , and the end load of the arm was $5 \mathrm{~kg}$. The number of uniform discrete pose in the workspace point was taken as follows: $N_{p}=40$. Then, the TOSM of the robot system at each workspace point was solved through combining with the relevant parameters of the robot, and the TOSM of each workspace point in its workspace is drawn, as shown in Figure 6 .

Each ball represents a workspace point of the arm, and the color of the ball reflects the size of the TOSM at this point. The maximum tip-over stability margin is 0.91 , the minimum is 0.69 , and the average is 0.80 from the simulation results. In addition, it can be seen that the shape of the discrete space is not a sphere, but a flat shape in the $y$ axis direction, which is due to the particularity of the modular manipulator configuration. There is little difference in the $\psi$ value in the static workspace from Figure 6. When the position of the arm end is farther away from the origin of the base coordinate, the value of $\psi$ becomes smaller and smaller, that is, the stability of the robot system is worse. From the sectional view, it can be seen that the value of $\psi$ close to the origin of the coordinate system is larger, that is, the better the stability of the robot.

5.2. Joint Speeds' Case. The purpose of considering joint speeds is to investigate the effect of the coupling term due to centrifugal forces and gyroscopic moments on tip-over stability. In this section, all joint accelerations are set to zero. There are many kinds of joint speeds of the arm in any 


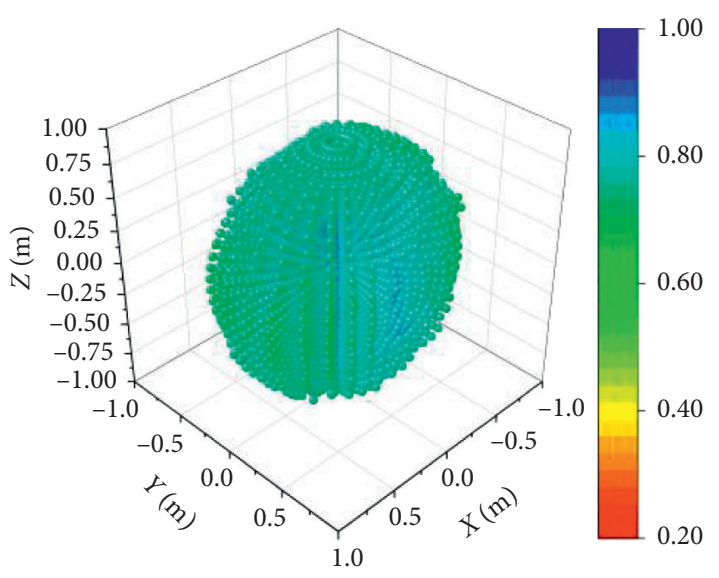

(a)

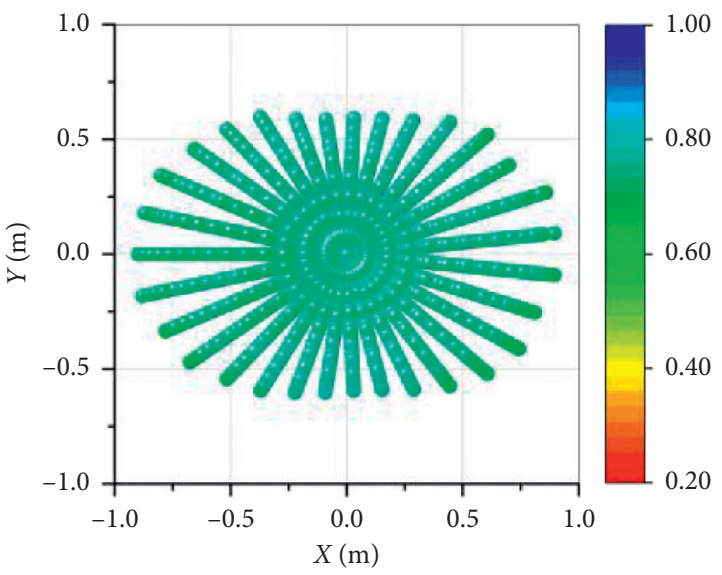

(c)

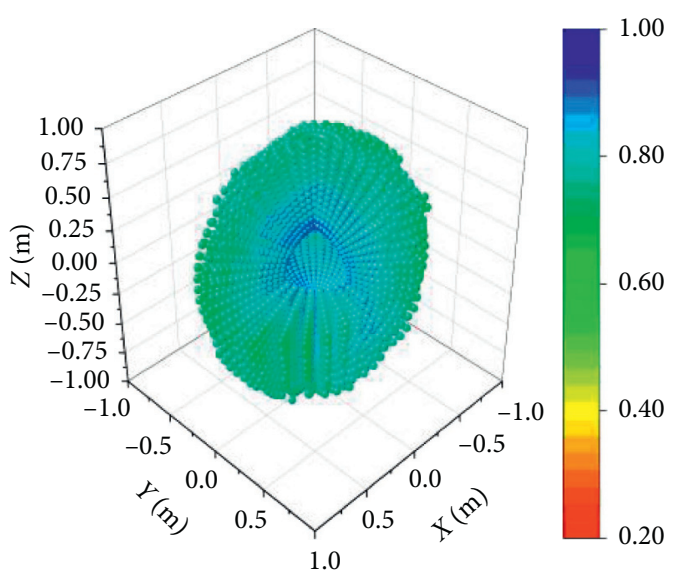

(b)

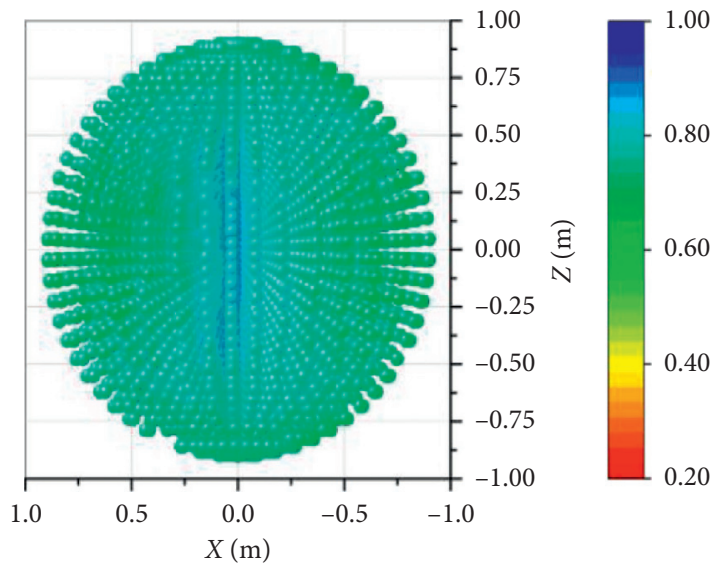

(d)

Figure 6: TOSM of the system in the workspace under the static condition. (a) Axonometric view. (b) Section view. (c) $X$ - $Y$ plane. (d) $X-Z$ plane.

possible configuration of the arm. In order to find out the situation that has a great impact on the tip-over stability of the robot system, the configuration of the arm was calculated, as shown in Table 2. The speed of the first three joints was set to change from $-\pi / 2$ to $\pi / 2$, at the same time, and the load at the end of the arm was $5 \mathrm{~kg}$, and then, the change of system TOSM with angular velocity can be obtained, as shown in Figure 7.

It can be seen from Figure 7 that the TOSM values in the forward and reverse directions are symmetrical under the three configurations, and when the joint speed is 0, the TOSM values of the three configurations are the largest, that is, the system stability is the best. When the first three joint speeds of the arm are at the maximum value in the forward direction or the maximum value in the reverse direction, the TOSM of the robot system is the minimum, that is, the stability of the robot system is the worst. Therefore, the maximum value of joint speed was considered, that is, the case of the first three joints with $\pi / 2 \mathrm{rad} / \mathrm{s}$.

The joint speed of the first three joints of the manipulator was set as $\dot{\theta}_{1}=\pi / 2 \mathrm{rad} / \mathrm{s}, \dot{\theta}_{2}=\pi / 2 \mathrm{rad} / \mathrm{s}$, and $\dot{\theta}_{3}=\pi / 2 \mathrm{rad} / \mathrm{s}$, while the joint speed of the last three joints was 0 . Then, the robot system TOSM of each workspace point can be
TABLE 2: Different configurations of the manipulator.

\begin{tabular}{lc}
\hline Arm configuration & Joint angle $(\mathrm{rad})$ \\
\hline 1 & {$[\pi / 2, \pi / 2, \pi / 2,0,0,0]$} \\
2 & {$[\pi / 3, \pi / 3, \pi / 3,0,0,0]$} \\
3 & {$[\pi / 6, \pi / 6, \pi / 6,0,0,0]$} \\
\hline
\end{tabular}

obtained, and the TOSM of arm workspace was drawn, as shown in Figure 8.

The maximum TOSM is 0.83 and the minimum is 0.22 from Figure 8. The TOSM in the whole workspace is greater than 0 , which means that the robot is always in a stable state. The difference between the maximum value and the minimum value is 0.61 from the color distribution. Moreover, when the position is farther away from the origin of the base coordinates on the $X-Y$ plane, the TOSM tends to be smaller, that is, the stability of the robot is the worse. It can be seen from the sectional view that the TOSM in the area close to the origin is relatively large, that is, the stability of the robot system is better.

5.3. Joint Accelerations' Case. The purpose of considering joint accelerations is to investigate the effect of inertia forces 


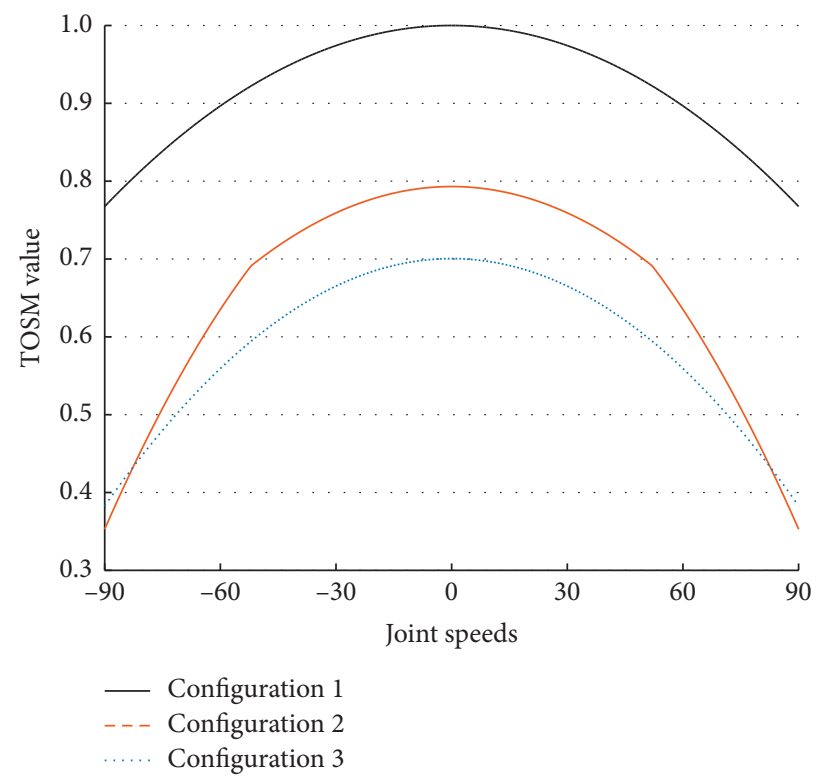

FIGURE 7: Variation of tip-over stability margin with joint speed in different configurations.

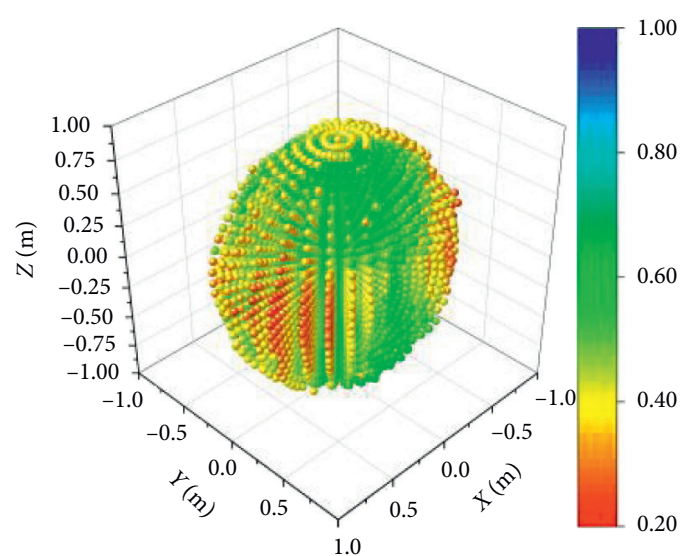

(a)

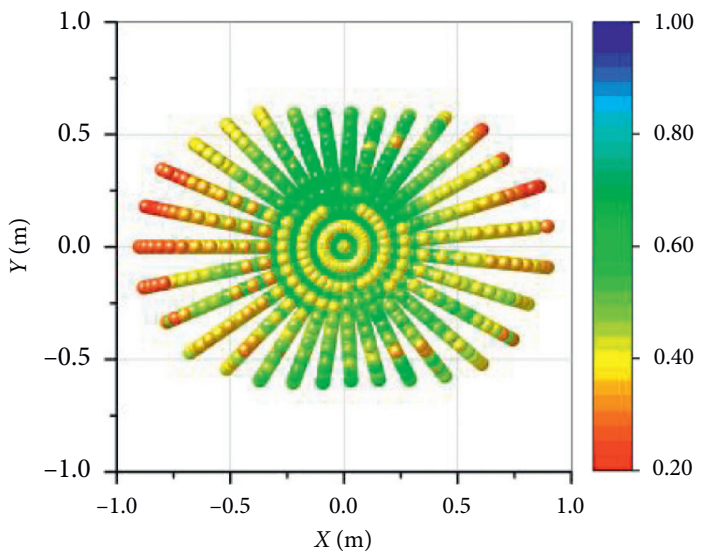

(c)

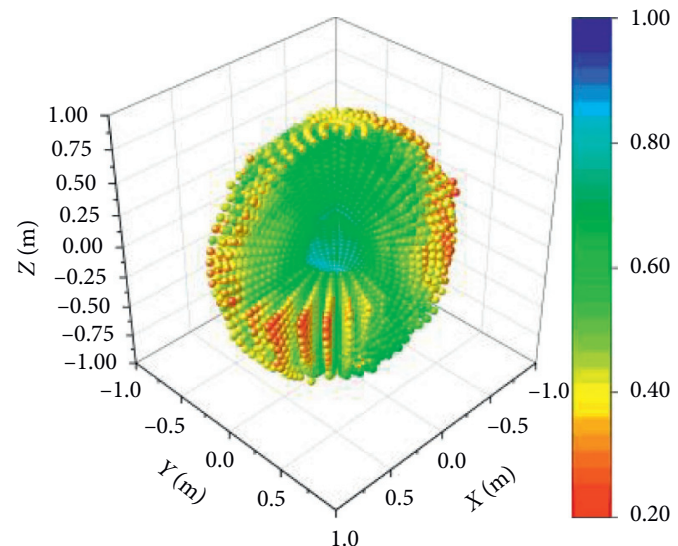

(b)

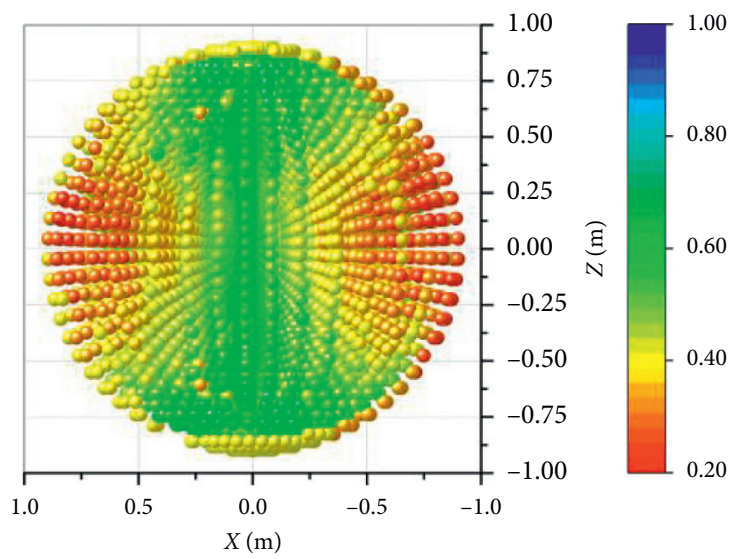

(d)

Figure 8: TOSM of the system in the workspace under the speed condition. (a) Axonometric view. (b) Section view. (c) $X$ - $Y$ plane. (d) $X-Z$ plane. 


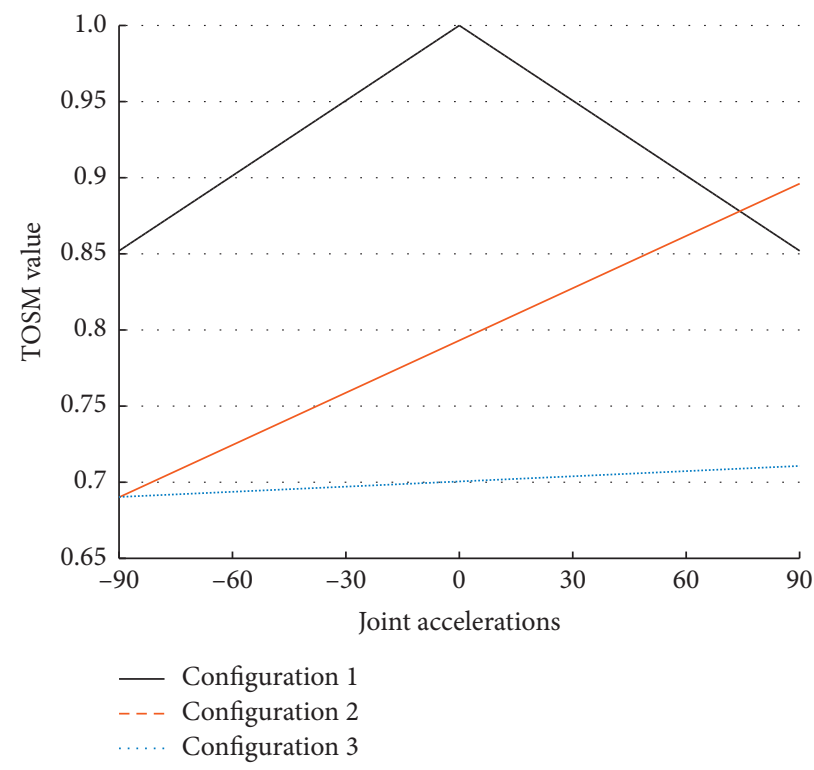

FIGURE 9: Variation of TOSM with joint acceleration in different configurations.

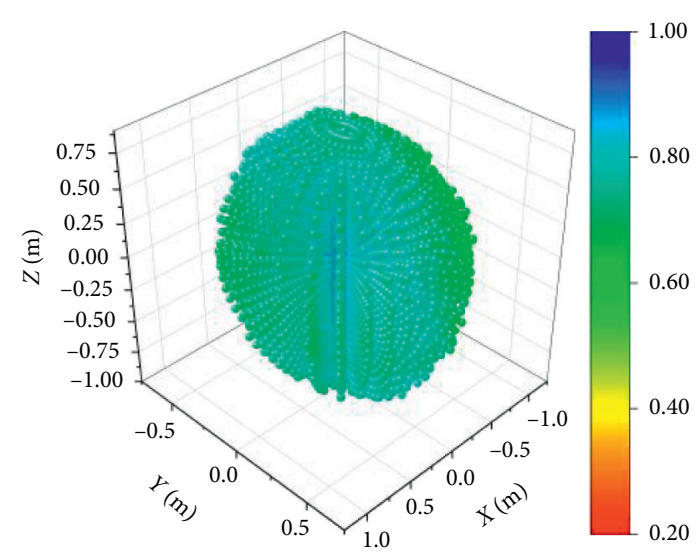

(a)

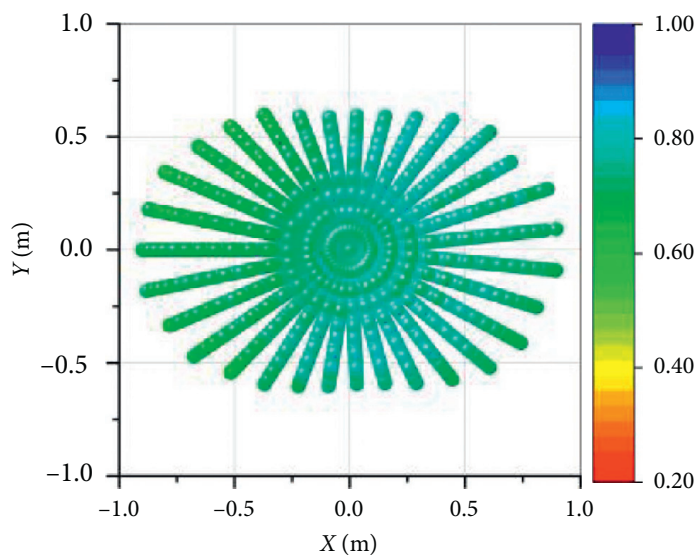

(c)

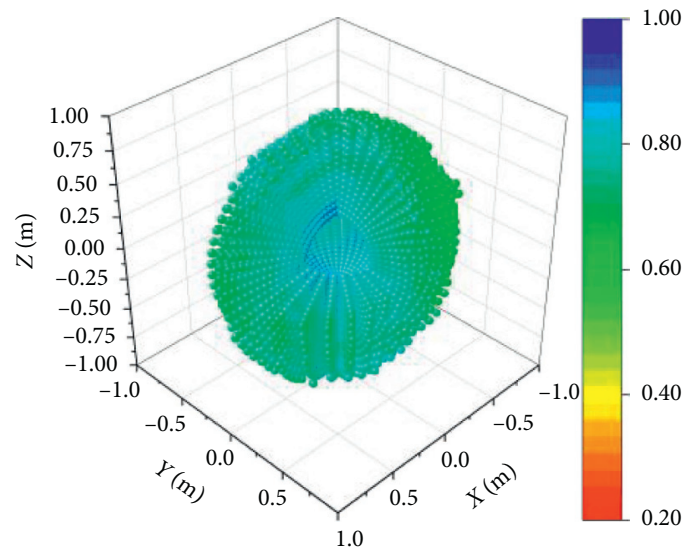

(b)

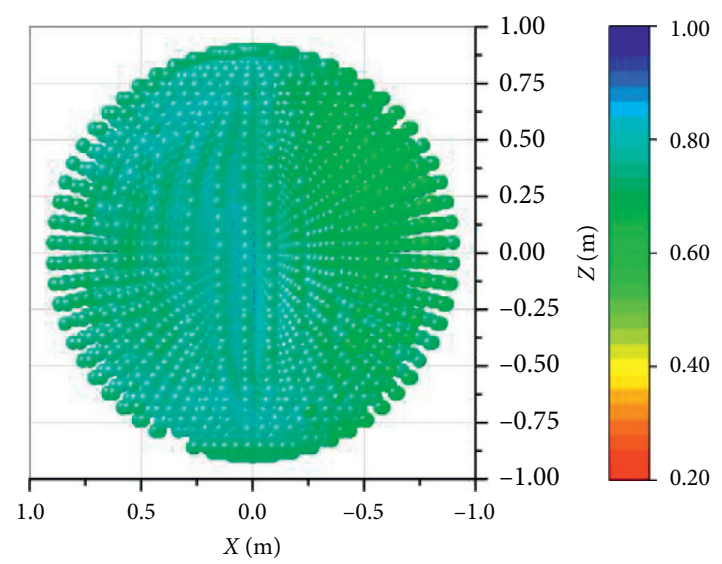

(d)

FIgUre 10: TOSM of the system in the workspace under the acceleration condition. (a) Axonometric view. (b) Section view. (c) $X$ - $Y$ plane. (d) $X-Z$ plane. 
TABle 3: Comparsion of three cases.

\begin{tabular}{lcccr}
\hline Situation & Maximum value & Minimum value & Average value & The difference between the two \\
\hline Static & 0.91 & 0.69 & 0.80 & 0.22 \\
Speed & 0.83 & 0.22 & 0.56 & 0.61 \\
Acceleration & 0.89 & 0.60 & 0.78 & 0.29 \\
\hline
\end{tabular}

and moments on tip-over stability. In this section, all joint speeds are set to zero as well. There are many kinds of accelerations of each joint in any possible configuration of the manipulator. In order to find out the situation that has a great impact on the tip-over stability of the robot system, the angular accelerations of the first three joints were set to change from $-\pi / 2 \mathrm{rad} / \mathrm{s}^{2}$ to $\pi / 2 \mathrm{rad} / \mathrm{s}^{2}$ at the same time; the three configurations of the arm are shown in Table 2 . Then, the change of TOSM with joint acceleration can be obtained, as shown in Figure 9.

It can be seen from Figure 9 that the TOSM of configuration 1 is symmetrically distributed in the forward and reverse directions, and the minimum value is obtained at $-\pi / 2 \mathrm{rad} / \mathrm{s}^{2}$ or $\pi / 2 \mathrm{rad} / \mathrm{s}^{2}$, and the stability of the system is the worst. However, configuration 2 and configuration 3 gradually increase with joint acceleration, and the worst stability of the system is at $-\pi / 2 \mathrm{rad} / \mathrm{s}^{2}$. Therefore, the accelerations of first three joints were set as $\ddot{\theta}_{1}=-\pi / 2 \mathrm{rad} / \mathrm{s}$, $\ddot{\theta}_{2}=-\pi / 2 \mathrm{rad} / \mathrm{s}$, and $\ddot{\theta}_{3}=-\pi / 2 \mathrm{rad} / \mathrm{s}$, while the joint acceleration of the last three joints was 0 . Then, the robot system TOSM of each workspace point can be obtained, and the TOSM was drawn, as shown in Figure 10.

The TOSM in the whole workspace is greater than 0 from Figure 10, which means that the robot system is always in a stable state. The maximum and minimum values of TOSM are 0.89 and 0.60 , respectively. The TOSM in the negative direction of the $X$-axis is smaller than that in the positive direction of $X$-axis from the color distribution, so the stability of the system is worse. In addition, it can be seen from the sectional view that the most stable region is still in the origin.

Comparing the results of the three cases, the TOSM data of the system are shown in Table 3.

It can be seen that, under the same load, the stability of the system is the worst under the speed cases, and the acceleration case is the second; the stability of the system under the static condition is the best.

\section{Conclusion}

In this paper, the force and moment of the arm have been analyzed by kinematics and dynamics, and an improved tipover moment stability criterion for a WMDAR system was presented. Then, a new method for calculating TOSM was derived based on the improved tip-over moment stability criterion for tip-over stability analysis, and three cases were simulated. The static, joint speed, and joint acceleration cases were simulated with the conclusion that the end position of the whole arm, angular velocity, and angular acceleration determine the stability condition. The results show that the WMDAR system exhibits overall tip-over stability within the given range of static loads, joint speeds, and accelerations. This paper proposes a method to study the stability of WMDAR, and this algorithm is very important for the follow-up research. The dynamic stability of the robot can be calculated by integrating the algorithm into the control elements, which lay a foundation for the trajectory planning of the robot to tip-over avoidance.

\section{Data Availability}

The data used to support the findings of the study are included within the paper.

\section{Conflicts of Interest}

The authors declare that they have no conflicts of interest.

\section{Acknowledgments}

This work was supported by the Opening Project of Shanghai Robot R\&D and Transformation Functional Platform (K2020468), Graduate Core (First-Class) Curriculum Construction Project of Anhui University of Science and Technology (2020HX010), and National Natural Science Foundation of China (61803251) and in part by the Graduate Innovation Fund of Anhui University of Science and Technology (2020CX2042).

\section{References}

[1] J. Zhu, Y. Pei, Y. Liu, and D. Gao, "Design and implementation of dual-arm mobile robot system based on wireless transmission," in Proceedings of the International Conferaence on Computer and Communication Systems, pp. 757-760, Shanghai, China, May 2020.

[2] P. D. Lillo, F. Pierri, F. Caccavale, and G. Antonelli, "Experiments on whole-body control of a dual-arm mobile robot with the set-based task-priority inverse kinematics algorithm," in Proceedings of the IEEE/RSJ International Conference on Intelligent Robots and Systems, pp. 9096-9101, Las Vegas, NV, USA, January 2020.

[3] C. J. Conti, A. S. Varde, and W. Wang, "Robot action planning by commonsense knowledge in human-robot collaborative tasks," in Proceedings of the IEEE International IOT, Electronics and Mechatronics Conference, pp. 170-176, Vancouver, Canada, September 2020.

[4] T. H. S. Li, Y. F. Ho, P. H. Kuo, Y. T. Ye, and L.-F. Wu, "Natural walking reference generation based on double-link LIPM gait planning algorithm," IEEE Access, vol. 5, pp. 2459-2469, 2017.

[5] M. B. Cheng, W. C. Su, C. C. Tsai, and T. Nguyen, "Intelligent tracking control of a dual-arm wheeled mobile manipulator with dynamic uncertainties," International Journal of Robust and Nonlinear Control, vol. 23, no. 8, pp. 839-857, 2013. 
[6] S. Caron, Q. C. Pham, and Y. Nakamura, "ZMP support areas for multicontact mobility under frictional constraints," IEEE Transactions on Robotics, vol. 33, no. 1, pp. 67-80, 2017.

[7] S. Sugano, Q. Huang, and I. Kato, "Stability criteria in controlling mobile robotic systems," in Proceedings of the IEEE/ RSJ International Conference on Intelligent Robots and Systems, pp. 832-838, Yokohama, Japan, July 1993.

[8] M. H. Korayem, V. Azimirad, A. Nikoobin, and Z. Boroujeni, "Maximum load-carrying capacity of autonomous mobile manipulator in an environment with obstacle considering tip over stability," International Journal of Advanced Manufacturing Technology, vol. 46, pp. 811-829, 2010.

[9] S. Kagami, T. Kitagawa, K. Nishiwaki, T. Sugihara, M. Inaba, and $\mathrm{H}$. Inoue, "A fast dynamically equilibrated walking trajectory generation method of humanoid robot," Autonomous Robots, vol. 12, no. 1, pp. 71-82, 2002.

[10] E. G. Papadopoulos and D. A. Rey, "A new measure of tipover stability margin for mobile manipulators," in Proceedings of the IEEE International Conference on Robotics and Automation, pp. 3111-3116, Minneapolis, MN, USA, April 1996.

[11] D. A. Rey and E. G. Papadopoulos, "Online automatic tipover prevention for mobile manipulators," in Proceedings of the IEEE/RSJ International Conference on Intelligent Robots and Systems, pp. 1273-1278, Grenoble, France, September 1997.

[12] S. A. A. Moosavian and K. Alipour, "Moment-height tip-over measure for stability analysis of mobile robotic systems," in Proceedings of the IEEE International Conference on Intelligent Robots and Systems, pp. 5546-5551, Beijing, China, October 2006.

[13] S. A. A. Moosavian and K. Alipour, "Tip-over stability of suspended wheeled mobile robots," in Proceedings of the IEEE International Conference on Mechatronics and Automation, pp. 1356-1361, Harbin, China, August 2007.

[14] S. A. A. Moosavian and K. Alipour, "On the dynamic tip-over stability of wheeled mobile manipulators," International Journal of Robotics and Automation, vol. 22, no. 4, pp. 322328, 2007.

[15] K. Alipour and S. A. A. Moosavian, "Postural stability of wheeled mobile manipulators with flexible suspension considering tire friction model," in Proceedings of the IEEE/ASME International Conference on Advanced Intelligent Mechatronics, pp. 764-769, Singapore, July 2009.

[16] A. Ghasempoor and N. Sepehri, "A measure of machine stability for moving base manipulators," in Proceedings of the IEEE International Conference on Robotics and Automation, pp. 2249-2254, Nagoya, Japan, May 1995.

[17] S. Guo, T. Song, F. Xi, and R. P. Mohamed, “Tip-over stability analysis for a wheeled mobile manipulator," Journal of Dynamic Systems, Measurement, and Control, vol. 139, no. 5, 2017.

[18] Y. Liu and G. Liu, "Interaction analysis and online tip-over avoidance for a reconfigurable tracked mobile modular manipulator negotiating slopes," IEEE/ASME Transactions on Mechatronics, vol. 15, no. 4, pp. 623-635, 2010.

[19] P. Moubarak and P. Ben-Tzvi, "A globally converging algorithm for adaptive manipulation and trajectory following for mobile robots with serial redundant arms," Robotica, vol. 31, no. 8, pp. 1299-1311, 2013.

[20] Y. Li and Y. Liu, "Real-time tip-over prevention and path following control for redundant nonholonomic mobile modular manipulators via fuzzy and neural-fuzzy approaches," Journal of Dynamic Systems, Measurement, and Control, vol. 128, no. 4, pp. 753-764, 2006.
[21] X. Ding, Y. Liu, J. Hou, and Q. Ma, “Online dynamic tip-over avoidance for a wheeled mobile manipulator with an improved tip-over moment stability criterion," IEEE Access, vol. 7, pp. 67632-67645, 2019.

[22] M. Agheli and S. Nestinger, "Foot force based reactive stability of multi-legged robots to external perturbations," Journal of Intelligent and Robotic Systems, vol. 81, no. 3-4, pp. 287-300, 2016.

[23] J. Feng, W. Zhang, and G. Guo, "Reseach on a new approach of auto self-balancing for rescue robot on uneven terrain," in Proceedings of the IEEE International Conference on Robotics and Biomimetics, pp. 74-79, Macau, China, December 2017.

[24] A. K. Kashyap and D. R. Parhi, "Optimization of stability of humanoid robot NAO using ant colony optimization tuned MPC controller for uneven path," Soft Computing, vol. 25, no. 7, pp. 5131-5150, 2021.

[25] R. M. Murray, Z. Li, and S. S. Sastry, A Mathematical Introduction to Robotic Manipulation, CRC Press, Boca Raton, FL, USA, 1994.

[26] E. B. Saff and A. B. J. Kuijlaars, "Distributing many points on a sphere," The Mathematical Intelligencer, vol. 19, no. 1, pp. 5-11, 1997. 